\title{
Study of non-contrast helical computed tomography in evaluating holmium laser lithotripsy for urinary calculus
}

\author{
JIA MI $^{1}$, ZUDONG YIN ${ }^{2}$, XINYI ZHANG ${ }^{2}$, WUSHI HAN ${ }^{2}$, XIANGSEN JIANG ${ }^{2}$, CHANGBIN WANG $^{2}$, \\ XIAOBAO $\mathrm{LI}^{2}$, ZHANGZHU LI ${ }^{2}$, LEI YU ${ }^{2}$, LIANG YIN ${ }^{2}$ and $\mathrm{LIN} \mathrm{CHENG}^{3}$ \\ ${ }^{1}$ Department of Ultrasound, Shandong Provincial Third Hospital; \\ ${ }^{2}$ CT Department, Medical Imaging Center, Shandong Provincial Third Hospital, Jinan, Shandong 250031; \\ ${ }^{3}$ Department of Ultrasound, Qilu Hospital of Shandong University, Jinan, Shandong 250012, P.R. China
}

Received March 13, 2018; Accepted July 20, 2018

DOI: $10.3892 /$ etm.2018.6765

\begin{abstract}
The present study aimed to investigate the correlation between the parameters of non-contrast helical computed tomography (NCHCT) and the total energy of holmium laser lithotripsy, and establish a correlative mathematical model. From March 2016 to February 2017, 120 patients with a single urinary calculus were examined by NCHCT prior to holmium laser lithotripsy. The calculus location was confirmed, the $\mathrm{CT}$ value was measured and the volume of the calculus in the established three-dimensional reconstruction model was calculated. The total energy of lithotripsy (TEL) was recorded post-operatively. A significant difference in the TEL between renal calculi and ureteral calculi was identified $(\mathrm{P}<0.001)$ and a high and significant correlation between the volume of the calculus and the TEL was determined (Spearman $r=0.827$, $\mathrm{P}<0.001)$. A moderate correlation was identified between the CT value of the calculus and the TEL (Spearman $r=0.468$, $\mathrm{P}<0.001)$. Multivariate linear regression analysis revealed that the location, the volume and the CT value of the calculus were independently associated with the TEL $(\mathrm{F}=288.858$, adjusted $\left.\mathrm{R}^{2}=0.879, \mathrm{P}<0.01\right)$. A mathematical model correlating the parameters of NCHCT with the TEL was established, which may provide a foundation to guide the use of energy in holmium laser lithotripsy, and it was possible to estimate the TEL by the location, the volume and the CT value of the calculus.
\end{abstract}

\section{Introduction}

Urinary calculus is commonly encountered in urinary surgery, and its worldwide incidence has been constantly increasing in recent years (1). The cause of urinary calculus is complex and

Correspondence to: Dr Lin Cheng, Department of Ultrasound, Qilu Hospital of Shandong University, 107 Wenhua Xi Road, Jinan, Shandong 250012, P.R. China

E-mail: lincheng77@126.com

Key words: non-contrast helical computed tomography, calculus, holmium laser lithotripsy the major influencing factors include individual and environmental factors. Urinary calculus is likely to cause urinary tract obstruction, accompanied by dull or colic pain in the waist, hematuria and fever, which severely affects the quality of life and the health of affected patients (2-4).

Non-contrast helical computed tomography (NCHCT) is the gold standard for the diagnosis of urinary calculus, and provides accurate information, including the size, location, shape and amount of calculi. NCHCT provides a preliminary estimation of the hardness and fragility of urinary calculi by measuring the computed tomography (CT) value, which is the X-ray absorption coefficient of a certain tissue/material, and the volume of the calculus may be accurately calculated through the establishment of a three-dimensional reconstruction model.

After the first successful application of holmium laser to the urinary calculus (5), this technique has received increasing attention. Due to its precise and powerful fragmenting function and high safety, holmium laser has become one of the most popular treatments for urinary lithotripsy. Holmium laser is able to fragment the calculus more effectively than extracorporeal shockwave and other lithotripsy, and achieve a high calculus-free rate of $>90 \%(6,7)$. Compared to other fragmenting equipment, holmium laser generates smaller fragments of the calculus, which are easier to discharge (8). Holmium laser treatment causes minimal injury to the surrounding area and is safer than other fragmenting operations (9).

Certain studies have demonstrated that the mechanism of holmium laser lithotripsy maybe a photomechanical effect. When the laser fiber is exposed to the calculus, the large energy is immediately absorbed by the internal and external water of the calculus. Cavitation bubbles are produced at the water-calculus interface and the shock waves produced by continuous rebound and countless bursting of the bubbles are transferred to the calculus, resulting in its fragmentation $(10,11)$. However, certain studies have refuted this view. Dushinski and Lingeman (12) proposed that the mechanism of fragmentation should be explained by the photothermal effect on urinary calculi. Chan et al (13) also reported that the major mechanism of holmium laser lithotripsy is the photothermal effect. The large energy of the holmium laser increases the temperature of the irradiated region and exceeds the threshold 
temperature. The heat causes a chemical breakdown of the calculus and weakens the structural integrity of the calculus, and it also contributes to the fragmentation of the interstitial water and vapor expansion.

Several parameters affecting the effect of fragmentation of calculi by holmium laser lithotripsy have been evaluated. Sea et al (14) reported that with the increase of the frequency of constant pulse energy, the crushing rate was not increased. Chawla et al (15) have reported that the crushing rate rises accompanied by the increase of the pulse energy, but it does not increase with the pulse frequency. Kronenberg and Traxer (16) indicated that the setting of a low pulse repetition frequency and high pulse energy achieved a higher crushing rate. Bader et al (7) suggested that with the same power setting, there was no significant difference in the fragmentation rate between different pulse durations. Furthermore, Kronenberg and Traxer (16) observed that at the same power level, a setting with a low frequency and high pulse energy is more efficient than high a frequency and low pulse energy. They also identified a linear correlation of the pulse energy with the size of the fragment, as well as with the width and depth of the fissure.

Previous theoretical and clinical studies on holmium laser lithotripsy mainly focused on the mechanism, influencing factors, applied range and therapeutic efficacy of the operation. Only a few studies have assessed the correlation between the parameters of NCHCT and the total energy of holmium laser lithotripsy (17). Few studies investigated the guidance of the energy use in holmium laser lithotripsy (18). The purpose of the present study was to investigate the correlation between the parameters of NCHCT and the total energy of lithotripsy (TEL), and to establish a correlative mathematical model.

\section{Materials and methods}

Patients. A total of 125 patients with urinary calculi who presented at Shandong Provincial Third Hospital (Jinan, China) between March 2016 and February 2017, and who were scheduled for holmium laser lithotripsy, were enrolled in the present study. The present study was approved by the institutional review board of Shandong Provincial Third Hospital. All patients provided written informed consent prior to enrollment. Of all of the patients, 5 were excluded from the final analyses due to failure of their lithotripsy: In three cases, the calculi in the subrenal calyx were too secluded to be reached and fragmented effectively, and two cases of residual fragments sized $>4 \mathrm{~mm}$ after the procedure were encountered. Therefore, the present study collected valid data from 120 patients. Of the 120 patients, 77 were female and 43 were male, the age range was $26-78$ years and the average age was $47.9 \pm 12.7$ years.

NCHCT examination. NCHCT examination was performed with a Philips Brilliance iCT 256 scanner (Philips Healthcare, Eindhoven, The Netherlands). All patients were examined one day prior to lithotripsy. The patients were fasted for at least $8 \mathrm{~h}$ to avoid any interference from intestinal gas and chyme on the results of the examination. The patients were requested to drink $500-1,000 \mathrm{ml}$ water at 1prior to the examination to moderately fill the bladder.

The patients were scanned in a supine position with their hands on their head, and were requested to hold their breath during the scan. Image archiving and communication systems were used to extract the CT images of the patients. First, the position of each calculus was determined and the calculus was classified as either a renal calculus and a ureteral calculus according to its position, and the number of calculi was recorded. Furthermore, the three-dimensional reconstruction model of the calculus was established based on the CT images capturedon an Extended Brilliance Workspace workstation (Philips Healthcare), and its volume was calculated. The detailed procedures were as follows: i) The calculus was manually colored, ii) the remnant was incised around the calculus manually, iii) the three-dimensional image of the calculus was reconstruct, iv) the calculus volume was calculated using a computer (Fig. 1). Third, the images were magnified by three-fold and the image of the calculus displaying the longest diameter was selected, in which the central region of $0.02 \mathrm{~cm}^{2}$ was selected as the region of interest and the CT value was measured. Another two adjacent images were used to measure the CT value of the region of interest (Fig. 2). The average value of the three measurements was used as the final CT value of the calculus. It was divided into four grades: 1, CT <400 Hounsfield units (Hu); 2, Ct=400-799 Hu; 3, $\mathrm{CT}=800-1,199 \mathrm{Hu} ; 4, \mathrm{CT} \geq 1,200 \mathrm{Hu}$.

Lithotripsy. All lithotripsy treatments were performed by two surgical urologists (one with 5 years of experience and the other with 7 years of experience), and with a dual-wavelength holmium laser therapeutic machine (Power Suite 80/100w; Lumenis Ltd., Yokneam, Israel). The laser settings were as follows: Excitation/emission wavelengths of the laser fiber at $200 / 365 \mu \mathrm{m}$ with an output energy of $0.5 / 0.6 \mathrm{~J}$ and a pulse repetition rate of $20 / 35 \mathrm{~Hz}$. During lithotripsy, the calculus was targeted and fragmented into pieces as small as possible. Taking the laser fiber as a frame of reference, all fragments sized $>4 \mathrm{~mm}$ were removed with a basket catheter. The total energy of the completed laser lithotripsy was recorded. The criterion for a completed laser lithotripsy was no residual fragment sized $>4 \mathrm{~mm}$, and smaller fragments were expected to be spontaneously excreted. The efficacy of the lithotripsy was evaluated 1-2 days after performing an ultrasound and kidney, ureter and bladder X-ray.

Statistical analysis. All statistical analyses were performed with the SPSS 17.0 software package (SPSS, Inc., Chicago, IL, USA). After the normality was determined by the Kolmogorov-Smirmnov test, Spearman's rank correlation analysis was used to assess the correlations between the TEL, and the location, the volume and the CT value of the calculi. Multivariate linear regression (forward selection method) was performed to formulate a mathematical model to estimate the TEL. $\mathrm{P}<0.05$ was considered to indicate a statistically significant difference.

\section{Results}

Overview of NCHCT. Concerning the calculus location, 48 patients $(40.0 \%)$ had renal calculi and $72(60.0 \%)$ had ureteral calculi. In terms of the calculus volume, the largest calculus was $1,347 \mathrm{~mm}^{3}$, the smallest was $254 \mathrm{~mm}^{3}$ and the average volume of the calculi was $485.35 \pm 195.349 \mathrm{~mm}^{3}$. The 


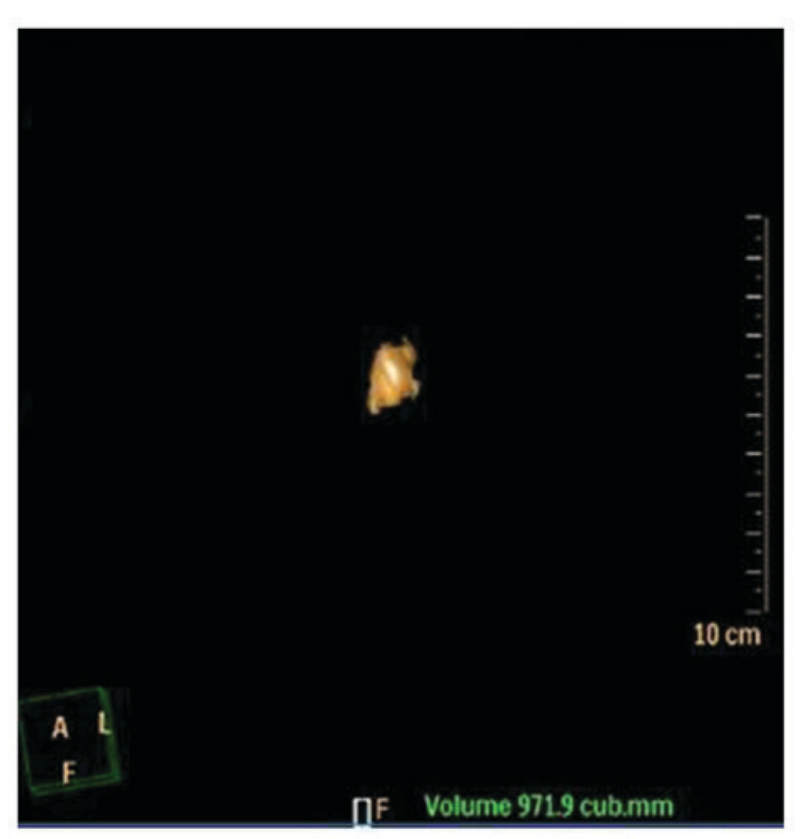

Figure 1. Three-dimensional reconstruction model of the calculus in a patient.

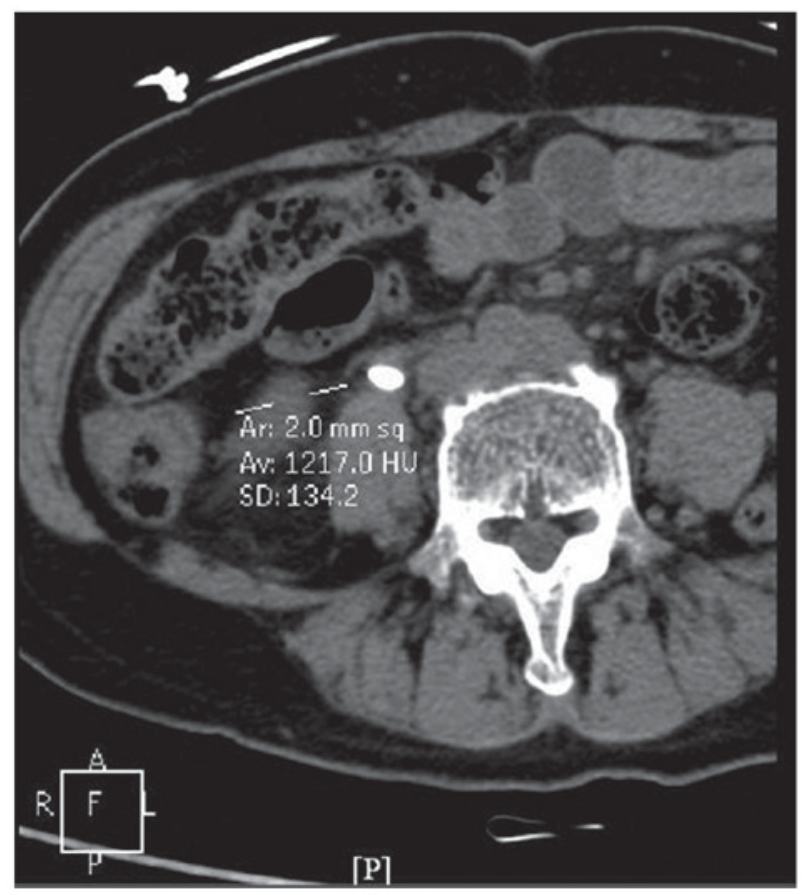

Figure 2. CT value of the calculus of a patient. Hu, Hounsfield units; SD, standard deviation; Av, average CT value; Ar, area; CT, computed tomography.

highest CT value was 1,475 $\mathrm{Hu}$ and the lowest CT value was 374 $\mathrm{Hu}$, and the average CT value of the calculi was $927.27 \pm 275.186$ $\mathrm{Hu}$. Among them, 5 calculi $(4.2 \%)$ were grade 1, $32(26.7 \%)$ were grade 2, $63(52.5 \%)$ were grade 3 and 20 (16.6\%) were grade 4 (Table I).

TEL correlates with the calculus location, volume and CT value. A strong negative correlation was identified between the TEL and calculus location $(\mathrm{r}=-0.819, \mathrm{P}<0.001)$; the TEL for the renal calculus was higher compared with the TEL for the ureteral
Table I. Parameters of non-contrast helical computed tomography.

\begin{tabular}{lc}
\hline Parameters & Value \\
\hline Calculus location & \\
Renal & $48(40)$ \\
Ureteral & $72(60)$ \\
CT value $(\mathrm{Hu})$ & $927.27 \pm 275.186$ \\
Grade & \\
$1(400 \mathrm{Hu})$ & $5(4.2)$ \\
$2(400-799 \mathrm{Hu})$ & $32(26.7)$ \\
$3(800-1,199 \mathrm{Hu})$ & $63(52.5)$ \\
$4(\geq 1,200 \mathrm{Hu})$ & $20(16.6)$ \\
Calculus volume $\left(\mathrm{mm}^{3}\right)$ & $485.35 \pm 195.349$ \\
\hline
\end{tabular}

Values are expressed as the mean \pm standard deviation or $\mathrm{n}(\%) . \mathrm{Hu}$, hounsfield units; CT, computed tomography.

calculus. There was a strong positive correlation between the volume of the calculus and the TEL ( $r=0.827, \mathrm{P}<0.001)$; larger calculus required higher TEL. A moderate correlation between the $\mathrm{CT}$ value of the calculus and the TEL was identified $(\mathrm{r}=0.468, \mathrm{P}<0.001)$; a calculus with a higher $\mathrm{CT}$ value required higher TEL. Multivariate linear regression analysis revealed that the location, the volume and the $\mathrm{CT}$ value of the calculus were independently associated with the TEL $(\mathrm{P}<0.01$; Table II).

Establishment of the mathematical model. To estimate the TEL of different calculi, a multivariate linear regression model was established with the following parameters: Calculus location, volume and CT value (Table III). After collinearity was eliminated and normality was tested (Figs. 3 and 4), the following multivariate linear regression equation was obtained: TEL $(\mathrm{J})=753.328-328.835 \mathrm{x}$ calculus location $(0=$ renal calculus; $1=$ ureteral calculus $)+0.940 \mathrm{x}$ calculus volume $\left(\mathrm{mm}^{3}\right)+0.421 \mathrm{x}$ $\mathrm{CT}$ value $(\mathrm{Hu})\left(\mathrm{F}=288.858\right.$, adjusted $\left.\mathrm{R}^{2}=0.879, \mathrm{P}<0.01\right)$. The adjusted $\mathrm{R}^{2}$ indicated that the energy variation based on the location, the volume and the $\mathrm{CT}$ value of the calculus accounted for $87.9 \%$ of the samples. The equation indicated that more energy was required for lithotripsy in patients with a renal calculus, a calculus with a larger volume and a calculus with a higher CT value.

\section{Discussion}

The TEL required to fragment calculi varies depending on their specific features. Unguided use of energy in lithotripsy has certain disadvantages. Insufficient energy may not fragment the calculus effectively, while excessive energy may lead to a higher incidence of complications and adverse effects. If the TEL was to be estimated pre-operatively, the urologist would be able to predict the difficulty of the operation and arrange for a suitable type of anesthesia, operation monitoring and drug treatment, and the emergency program for high-risk patientsmay also be performed in advance. To facilitate the pre-operativeestimation of the TEL, a mathematical model correlating the parameters 
Table II. Results of Spearman's rank correlation analysis of the parameters of NCHCT and the total energy of holmium laser lithotripsy.

\begin{tabular}{lcc}
\hline Parameters & $\mathrm{r}$ & P-value \\
\hline Calculus location & -0.819 & $<0.001$ \\
Calculus volume & 0.827 & $<0.001$ \\
CT Value & 0.468 & $<0.001$ \\
\hline
\end{tabular}

CT, computed tomography.

Table III. Results of multivariate linear regression analysis of the variables estimating the total energy of holmium laser lithotripsy.

\begin{tabular}{lrrrr}
\hline Variables & \multicolumn{1}{c}{ B } & SE & t & P-value \\
\hline Constant & 753.328 & 67.941 & 11.088 & $<0.001$ \\
Calculus location & -328.835 & 32.965 & -9.975 & $<0.001$ \\
Calculus volume & 0.940 & 0.084 & 11.244 & $<0.001$ \\
CT value & -0.421 & 0.045 & 9.373 & $<0.001$
\end{tabular}

CT, computed tomography; B, partial regression coefficient SE, error of partial regression coefficient standard.

of NCHCT with the TEL was established in the present study, which may provide a foundation to guide the use of energy in holmium laser lithotripsy. The safety and efficiency of laser lithotripsy may be improved by preliminary estimation of the total energy required for holmium laser lithotripsy.

The present study indicated that the TEL required for renal calculi exhibited a significant difference from that required for ureteral calculi, with renal calculi requiring a higher TEL. A previous retrospective study by Molina et al (17) indicated that renal calculi required more energy than ureteral calculi, which was in agreement with the results of the present study. Although the exact reasons for this remain elusive, one conceivable explanation is that hydronephrosis or calyceal hydrocalycosis make the renal calculus more mobilized, while the location of ureteral calculus is relatively fixed. In the fragmentation of a renal calculus, the impact produced by holmium laser may cause the movement of the calculus, and the total impact of the laser fiber on the calculus is reduced. As a result, more pulses may be fired inefficiently and more energy is wasted.

In the present study, a strong correlation between the calculus volume and the TEL was identified, with larger calculi requiring more energy for fragmentation. In their retrospective study, Molina et al (17) determined a significant correlation between the calculus volume and the cumulative holmium laser energy. Blomley et al (19) performed a systematic review of holmium laser lithotripsy and reported that the required cumulative energy of lithotripsy was increased with the increase of the calculus size and mass. These results are consistent with the conclusion of the present study and it was possible to evaluate the TEL preferably by the volume of the calculus, which may serve as an important index of the evaluation of the TEL.

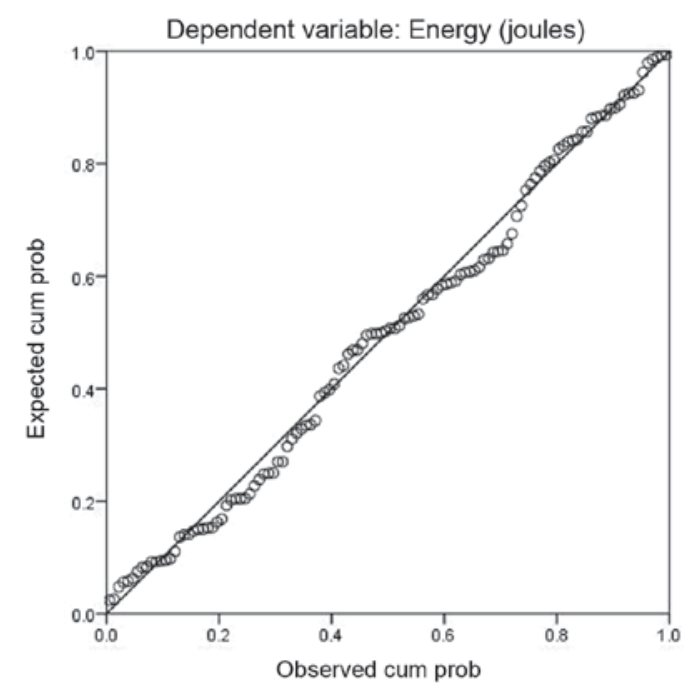

Figure 3. Normal P-P plot of regression-standardized residual. Cum prob, cumulative probability.

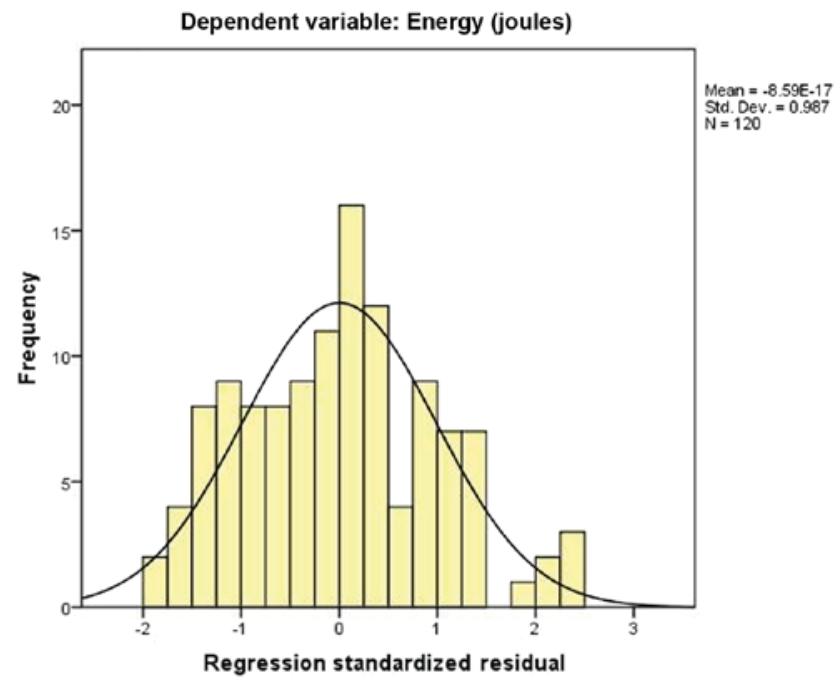

Figure 4. Histogram of regression standardized residual. Std. Dev., standard deviation.

The present study also determined a correlation between the CT value of the calculus and the TEL, with a calculus with a higher $\mathrm{CT}$ value requiring a higher TEL. Zhang et al (20) reported that the $\mathrm{CT}$ value was able to effectively predict the fragility of urinary calculi and the shocking times of extracorporeal shock wave lithotripsy. Gupta et al (21) came to the same conclusion that the fragility of a calculus may be estimated based on the CT value, with a lower CT value of the calculus being associated with an easier fragmentation. Wang et al (22) indicated that the $\mathrm{CT}$ value may be used to quantitatively analyze the hardness of urinary calculi. The study suggested a positive correlation between the $\mathrm{CT}$ value of urinary calculi and their hardness, with a higher $\mathrm{CT}$ value indicating a harder calculus and a more difficult fragmentation. The CT value is the X-ray absorption coefficient of a certain tissue/material, and it is an index providing information on the density, with a higher CT value indicating a larger density. However, the hardness and fragility of a urinary calculus mainly depends 
on its chemical composition and inner structure, which has a high correlation with the CT value and may be used to estimate the major composition of calculus. Furthermore, the hardness and fragility of the calculus have a key role in the efficacy of lithotripsy, which exhibits marked differences for calculi with different hardness and fragility. Overall, calculi with different CT values have a different hardness and fragility, and the TEL is different.

In conclusion, the present study indicated a correlation between the parameters of NCHCT and the TEL. A mathematical model correlating the parameters of NCHCT with the TEL was established, which may provide a foundation to guide the use of energy in holmium laser lithotripsy. By providing a preliminary evaluation of the total energy required in holmium laser lithotripsy, NCHCT may be used to predict the difficulty of lithotripsy, and improve the safety and efficiency of lithotripsy.

\section{Acknowledgements}

The authors are particularly grateful to Professor Yuanyuan Liu (Statistics Department, Shandong Provincial Third Hospital, Jinan, China) for her assistance with the statistical analysis. The authors also thank Professor Mingjie Li and Professor Xiangtao Wang (both Department of Urinary Surgery, Shandong Provincial Third Hospital) for their technical advice on the holmium laser lithotripsy.

\section{Funding}

The present study was funded by the Science Foundation of Qilu Hospital of Shandong University and the Fundamental Research Funds of Shandong University (grant no. 2015QLMS39).

\section{Availability of data and materials}

The analyzed data sets generated during the present study are available from the corresponding author on reasonable request.

\section{Authors' contributions}

JM, ZY, XZ, CW, XL, ZL, LYu and LYi were responsible for data collection. LC, JM, ZY, XJ and WH were responsible for statistical analysis. LC, JM, ZY, XZ and WH wrote the article. The final version of the manuscript has been read and approved by all authors, and each author believes that the manuscript represents honest work.

\section{Ethical approval and consent to participate}

The present study was approved by the institutional review board of Shandong Provincial Third Hospital (Jinan, China). All patients provided written informed consent prior to enrollment.

\section{Patient consent for publication}

Not applicable.

\section{Competing interests}

The authors declare that they have no competing interests.

\section{References}

1. Brikowski TH, Lotan Y and Pearle MS: Climate-related increase in the prevalence of urolithiasis in the United States. Proc Natl Acad Sci USA 105: 9841-9846, 2008.

2. Kang HW, Lee SK, Kim WT, Kim YJ, Yun SJ, Lee SC and Kim WJ: Natural history of asymptomatic renal stones and prediction of stone related events. J Urol 189: 1740-1746, 2013.

3. Schwarzenbach HR and Jenzer S: Diagnosis and management of suspected nephrolithiasis in a primary care setting. Praxis (Bern 1994) 101: 1187-1192, 2012 (In German).

4. Wang SJ, Mu XN, Zhang LY, Liu QY and Jin XB: The incidence and clinical features of acute kidney injury secondary to ureteral calculi. Urol Res 40: 345-348, 2012.

5. Bagley D and Erhard M: Use of the holmium laser in the upper urinary tract. Tech Urol 1: 25-30, 1995.

6. Lam JS, Greene TD and Gupta M: Treatment of proximal ureteral calculi: Holmium:YAG laser ureterolithotripsy versus extracorporeal shock wave lithotripsy. J Urol 167: 1972-1976, 2002.

7. Bader MJ, Pongratz T, Khoder W, Stief CG, Herrmann T, Nagele U and Sroka R: Impact of pulse duration on Ho:YAG laser lithotripsy: Fragmentation and dusting performance. World J Urol 33: 471-477, 2015.

8. Johnson DE, Cromeens DM and Price RE: Use of the holmium:YAG laser in urology. Lasers Surg Med 12: 353-363, 1992.

9. Kronenberg P and Traxer O: Update on lasers in urology 2014: Current assessment on holmium:Yttrium-aluminum-garnet (Ho:YAG) laser lithotripter settings and laser fibers. World J Urol 33: 463-469, 2015.

10. Larizgoitia I and Pons JM: A systematic review of the clinical efficacy and effectiveness of the holmium:YAG laser in urology. BJU Int 84: 1-9, 1999.

11. Takazawa R, Kitayama S and Tsujii T: Successful outcome of flexible ureteroscopy with holmium laser lithotripsy for renal stones $2 \mathrm{~cm}$ or greater. Int J Urol 19: 264-267, 2012.

12. Dushinski JW and Lingeman JE: High-speed photographic evaluation of holmium laser. J Endourol 12: 177-181, 1998.

13. Chan KF, Vassar GJ, Pfefer TJ, Teichman JM, Glickman RD, Weintraub ST and Welch AJ: Holmium:YAG laser lithotripsy: A dominant photothermal ablative mechanism with chemical decomposition of urinary calculi. Lasers Surg Med 25: 22-37, 1999.

14. Sea J, Jonat LM, Chew BH, Qiu J, Wang B, Hoopman J, Milner T and Teichman JM: Optimal power settings for Holmium:YAG lithotripsy. J Urol 187: 914-919, 2012.

15. Chawla SN, Chang MF, Chang A, Lenoir J and Bagley DH: Effectiveness of high-frequency holmium:YAG laser stone fragmentation: The 'popcorn effect'. J Endourol 22: 645-650, 2008.

16. Kronenberg P and Traxer O: In vitro fragmentation efficiency of holmium: Yttrium-aluminum-garnet (YAG) laser lithotripsy-a comprehensive study encompassing different frequencies, pulse energies, total power levels and laser fibre diameters. BJU Int 114: 261-267, 2014.

17. Molina WR, Marchini GS, Pompeo A, Sehrt D, Kim FJ and Monga M: Determinants of Holmium:Yttrium-aluminum-garnet laser time and energy during ureteroscopic laser lithotripsy. Urology 83: 738-744, 2014.

18. Mi J, Li J, Zhang Q, Wang X, Liu H, Cao Y, Liu X, Sun X, Shang M and Liu Q: Combining ultrasonography and noncontrast helical computerized tomography to evaluate Holmium laser lithotripsy. Medicine (Baltimore) 95: e5564, 2016.

19. Blomley MJ, Nicholson DA, Bartal G, Foster C, Bradley A, Myers M, Man W, Li S and Banks LM: Holmium-YAG laser for gall stone fragmentation: An endoscopic tool. Gut 36: 442-445, 1995.

20. Zhang P, Tu B, Zhang J,Zhang He, Jiang H and Jiang N: Forecasting accuracy of dual-energy CT on kidney calculus components. 33: 636-638, 2011. (In Chinese).

21. Gupta NP,Ansari MS, Kesarvani P,Kapoor A and Mukhopadhyay S: Role of computed tomography with no contrast medium enhancement in predicting the outcome of extracorporeal shock wave lithotripsy for urinary calculi. BJU Int 95: 1285-1288, 2005.

22. Jinfeng Wang ZW, Xinguo Liu and Xiaogang Li: Prediction of hardness of urinary calculi by computed tomography in vitro. J Mod Urol 03: 191-193, 2008. (In Chinese). 